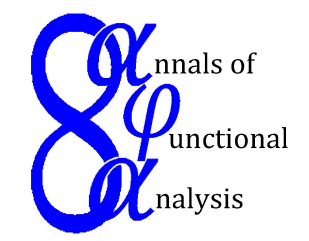

Ann. Funct. Anal. 6 (2015), no. 2, 116-132

http://doi.org/10.15352/afa/06-2-11

ISSN: 2008-8752 (electronic)

http://projecteuclid.org/afa

\title{
APPLICATIONS OF AN ANALOGUE OF CONDITIONAL WIENER INTEGRALS
}

\author{
IL YONG LEE, HYUN SOO CHUNG AND SEUNG JUN CHANG* \\ Communicated by C. Cuevas
}

ABSTRACT. In this paper, we obtain formulas for the analogue of conditional Wiener integrals for the functional $F$ of the form

$$
F(x)=\exp \left\{\int_{0}^{T} V(x(t)) d t\right\}, \quad x \in C[0, T]
$$

where $V: \mathbb{R} \rightarrow \mathbb{R}$ is a potential function. We then apply this formula to obtain several integration formulas for the functionals involving various potential functions which is used in quantum mechanics and other physical theories.

\section{INTRODUCTION}

For $T>0$, let $C_{0}[0, T]$ denote the one-parameter Wiener space; i.e., the space of real-valued continuous functions $x$ on $[0, T]$ with $x(0)=0$. We consider the partial differential equation

$$
\frac{\partial}{\partial t} \psi(u, t)=\frac{1}{2 \lambda} \frac{\partial^{2}}{\partial u^{2}} \psi(u, t)-V(u) \psi(u, t)
$$

with the initial condition $\psi(u, 0)=\varphi(u)$, where $V: \mathbb{R} \rightarrow \mathbb{R}$ is a potential function. Many mathematicians have attempted to solve (1.1), which is known as the diffusion equation. One popular method involves solving the Wiener integral

$$
\int_{C_{0}[0, T]} \varphi\left(\lambda^{-1 / 2} x(T)\right) \exp \left\{-\int_{0}^{T} V\left(\lambda^{-1 / 2} x(s)\right) d s\right\} d W(x)
$$

Date: Received: May 23, 2014; Accepted: Jul. 18, 2014.

* Corresponding author.

2010 Mathematics Subject Classification. Primary 60J65; Secondary 28C20, 43A32.

Key words and phrases. Pöschl-Teller potential, analogue of conditional Wiener integral, simple formula, harmonic oscillator, double-well potential. 
for $\lambda>0$, where $W$ is the standard Wiener measure on $C_{0}[0, T]$. This integral is obtained from the Feynman-Kac formula $[1,2,10]$. In previous works $[7,9,11]$, the authors introduced Fourier-type functionals. In [7], the authors studied an approach to solving the equation (1.1) using Fourier-type functionals and equation (1.2). Many physical problems can be formulated in terms of the conditional Wiener integral of a functional of the form

$$
F(x)=\exp \left\{-\int_{0}^{T} V(x(t)) d t\right\}, \quad x \in C_{0}[0, T] .
$$

In other words, we can solve equation (1.2) by using the conditional Wiener integral of a functional of the form (1.3).

Let $C[0, T]$ be the space of all real-valued continuous functions on $[0, T]$ with the supremum norm. In $[18,19]$, the authors introduced an analogue of the Wiener measure $w_{\varphi}$ on $(C[0, T], \mathcal{B}(C[0, T]))$, using a probability measure $\varphi$ on $(\mathbb{R}, \mathcal{B}(\mathbb{R}))$. Furthermore, in $[5,6,17,20]$, the authors studied various topics pertaining to this analogue of the Wiener measure, together with related topics concerning an analogue of Wiener space. In particular, the authors derived a simple formula for the conditional $w_{\varphi}$-integral and then used this formula to evaluate the conditional $w_{\varphi}$-integral of various functionals $[3,16]$.

In this paper, we introduce three functionals involving potential functions used in quantum mechanics. Functionals such as these have appeared in many papers $[8,14,15,21]$. We then evaluate the analogue of conditional Wiener integral of the functionals such as these. In Section 3, we derive a series representation of an analogue of conditional Wiener integral for the functional $F$ given by (1.3). In Section 4, we express the analogue of conditional Wiener integral ' $\mathrm{n}$ ' terms of the potential function $V(u)=u^{k}(k \in \mathbb{N})$. We then evaluate the analogue of conditional Wiener integral for functionals that involve potential functions.

\section{DEFINITIONS AND PRELIMINARIES}

In this section, we list some definitions and properties from $[3,4,5,6,16,17$, 18, 19, 20].

For $\vec{t}=\left(t_{0}, t_{1}, \cdots, t_{n}\right)$ with $0=t_{0}<t_{1}<\cdots<t_{n} \leq T$, let $J_{\vec{t}}: C[0, T] \rightarrow \mathbb{R}^{n+1}$ be the function given by

$$
J_{\vec{t}}(x)=\left(x\left(t_{0}\right), x\left(t_{1}\right), \cdots, x\left(t_{n}\right)\right) .
$$

For $B_{j}(j=0,1,2, \cdots, n)$ in $\mathcal{B}(\mathbb{R})$, the subset $J_{\vec{t}}^{-1}\left(\prod_{j=0}^{n} B_{j}\right)$ of $C[0, T]$ is called an interval and let $\mathcal{I}$ be the set of all such intervals. For a probability measure $\varphi$ on $(\mathbb{R}, \mathcal{B}(\mathbb{R}))$, let

$$
m_{\varphi}\left(J_{\vec{t}}^{-1}\left(\prod_{j=0}^{n} B_{j}\right)\right)=\int_{B_{0}} \int_{\prod_{j=1}^{n} B_{j}} W_{n+1}\left(\vec{t} ; u_{0}, u_{1}, \cdots, u_{n}\right) d\left(u_{1}, \cdots, u_{n}\right) d \varphi\left(u_{0}\right)
$$

where

$$
W_{n+1}\left(\vec{t} ; u_{0}, u_{1}, \cdots, u_{n}\right)=\left(\prod_{j=1}^{n} \frac{1}{2 \pi\left(t_{j}-t_{j-1}\right)}\right)^{\frac{1}{2}} \exp \left\{-\sum_{j=1}^{n} \frac{\left(u_{j}-u_{j-1}\right)^{2}}{2\left(t_{j}-t_{j-1}\right)}\right\} .
$$


By Theorem 5.1 [12, p.144] and Theorem 2.1 [12, p.212], the set of all Borel subsets in $C[0, T]$, coincides with the smallest $\sigma$-algebra generated by $\mathcal{I}$ and there exists a unique probability measure $w_{\varphi}$ on $\mathcal{B}(C[0, T])$ such that $w_{\varphi}(I)=m_{\varphi}(I)$ for all $I$ in $\mathcal{I}$. This measure $w_{\varphi}$ is called an analogue of Wiener measure associated with the probability measure $\varphi$ and the space $\left(C[0, T], \mathcal{B}(C[0, T]), w_{\varphi}\right)$ is called an analogue of Wiener space.

We denote the analogue of Wiener integral of a $\mathcal{B}(C[0, T])$-measurable function $F$ by

$$
E[F]=\int_{C[0, T]} F(x) d w_{\varphi}(x)
$$

whenever the integral exists.

The following formula follows easily from the Change of Variables Theorem.

Let $f: \mathbb{R}^{n+1} \rightarrow \mathbb{C}$ be a Borel measurable function. Then

$$
\begin{aligned}
& \int_{C[0, T]} f\left(x\left(t_{0}\right), x\left(t_{1}\right), \cdots, x\left(t_{n}\right)\right) d w_{\varphi}(x) \\
& \stackrel{*}{=} \int_{\mathbb{R}}\left[\int_{\mathbb{R}^{n}} f\left(u_{0}, u_{1}, \cdots, u_{n}\right) W_{n+1}\left(\vec{t} ; u_{0}, u_{1}, \cdots, u_{n}\right) d \prod_{j=1}^{n} u_{j}\right] d \varphi\left(u_{0}\right)
\end{aligned}
$$

where $d \prod_{j=1}^{n} u_{j}=d u_{1} \cdots d u_{n}$ and $\stackrel{*}{=}$ means that if either side exists, then both sides exist and they are equal. Then the equation (2.1) is reduced to the wellknown Wiener integration formula if $\varphi$ is the Dirac measure concentrated at 0 . For a more detailed study of this, see [18, 19].

We conclude this section with some simple examples of the analogue of Wiener integral.

Example 2.1. For $0 \leq t \leq T$, let $F(x)=\exp \{x(t)\}$. Then

$$
\int_{C[0, T]} \exp \{x(t)\} d w_{\varphi}(x)=\int_{\mathbb{R}} \exp \left\{u_{0}+\frac{t}{2}\right\} d \varphi\left(u_{0}\right) .
$$

If $\varphi$ is the Dirac measure $\delta_{0}$ at the origin in $\mathbb{R}$, then

$$
\int_{C[0, T]} \exp \{x(t)\} d w_{\varphi}(x)=\exp \left\{\frac{t}{2}\right\} .
$$

Hence, in this case the analogue of Wiener integral is equal to Wiener integral. If $\varphi$ has a normal distribution with mean $\alpha$ and variance $\sigma^{2}$, then

$$
\begin{aligned}
& \int_{C[0, T]} \exp \{x(t)\} d w_{\varphi}(x)=\int_{\mathbb{R}} \exp \left\{u_{0}+\frac{t}{2}\right\} d \varphi\left(u_{0}\right) \\
& =\frac{1}{\sqrt{2 \pi \sigma^{2}}} \int_{\mathbb{R}} \exp \left\{u_{0}+\frac{t}{2}\right\} \exp \left\{-\frac{\left(u_{0}-\alpha\right)^{2}}{2 \sigma^{2}}\right\} d u_{0}=\exp \left\{\frac{\sigma^{2}+t}{2}+\alpha\right\} .
\end{aligned}
$$

Example 2.2. Let $\mathcal{M}(\mathbb{R})$ denote the space of all complex-valued Borel measures on $\mathcal{B}(\mathbb{R})$. For $\nu \in \mathcal{M}(\mathbb{R})$, the Fourier-transform $\hat{\nu}$ of $\nu$ is defined by the formula

$$
\hat{\nu}(u)=\int_{\mathbb{R}} \exp \{i u v\} d \nu(v) .
$$


For $0 \leq t \leq T$, let $F(x)=\hat{\nu}(x(t))$. Then

$$
\int_{C[0, T]} \hat{\nu}(x(t)) d w_{\varphi}(x)=\int_{\mathbb{R}}\left[\int_{\mathbb{R}} \exp \left\{-\frac{t v^{2}}{2}+i u_{0} v\right\} d \varphi\left(u_{0}\right)\right] d \nu(v) .
$$

If $\varphi$ is a Dirac measure $\delta_{0}$ at the origin in $\mathbb{R}$, then

$$
\int_{C[0, T]} \hat{\nu}(x(t)) d w_{\varphi}(x)=\int_{\mathbb{R}} \exp \left\{-\frac{t v^{2}}{2}\right\} d \nu(v) .
$$

Once again, it can be concluded that the analogue of Wiener integral is equal to the Wiener integral, as described above. If $\varphi$ has a normal distribution with mean $\alpha$ and variance $\sigma^{2}$, then

$$
\begin{aligned}
& \int_{C[0, T]} \hat{\nu}(x(t)) d w_{\varphi}(x)=\int_{\mathbb{R}}\left[\int_{\mathbb{R}} \exp \left\{-\frac{t v^{2}}{2}+i u_{0} v\right\} d \varphi\left(u_{0}\right)\right] d \nu(v) \\
& =\frac{1}{\sqrt{2 \pi \sigma^{2}}} \int_{\mathbb{R}}\left[\int_{\mathbb{R}} \exp \left\{-\frac{t v^{2}}{2}+i u_{0} v\right\} \exp \left\{-\frac{\left(u_{0}-\alpha\right)^{2}}{2 \sigma^{2}}\right\} d u_{0}\right] d \nu(v) \\
& =\int_{\mathbb{R}} \exp \left\{-\frac{\left(t+\alpha^{2}\right)}{2} v^{2}+i \alpha v\right\} d \nu(v) .
\end{aligned}
$$

\section{An ANALOGUE OF CONDITIONAL WiENER INTEGRAL}

In this section, we derive a formula for the analogue of conditional Wiener integral for functionals $F$ of the form

$$
F(x)=\exp \left\{\int_{0}^{T} V(x(t)) d t\right\}, \quad x \in C[0, T]
$$

where $V: \mathbb{R} \rightarrow \mathbb{R}$ is a potential function.

First, we define the analogue of conditional Wiener integral.

Let $X$ be a $\mathbb{R}^{n+1}$-valued function on $C[0, T]$ whose probability distribution $w_{\varphi} \circ X^{-1}$ is absolutely continuous with respect to Lebesgue measure on $\mathbb{R}^{n+1}$. Let $F$ be a $\mathbb{C}$-valued $w_{\varphi}$-integrable functional on $C[0, T]$. Then an analogue of conditional Wiener integral of $F$ given $X$, denoted by $E[F \mid X](\vec{\eta})$, is a Lebesgue measurable function of $\vec{\eta}$, unique up to null sets in $\mathbb{R}^{n+1}$, satisfying the equation

$$
\int_{X^{-1}(B)} F(x) d w_{\varphi}(x)=\int_{B} E[F \mid X](\vec{\eta}) d\left(w_{\varphi} \circ X^{-1}\right)(\vec{\eta})
$$

for all $B \in \mathcal{B}\left(\mathbb{R}^{n+1}\right)$.

For each partition $\tau=\left\{t_{0}, t_{1}, \cdots, t_{n}\right\}$ of $[0, T]$ with $0=t_{0}<t_{1}<\cdots<t_{n}=T$, let $X_{\tau}: C[0, T] \rightarrow \mathbb{R}^{n+1}$ be defined by

$$
X_{\tau}(x)=\left(x\left(t_{0}\right), x\left(t_{1}\right), \cdots, x\left(t_{n}\right)\right) .
$$

Define a function $[x]$ on $[0, T]$ by

$$
[x](t)=x\left(t_{j-1}\right)+\frac{t-t_{j-1}}{t_{j}-t_{j-1}}\left(x\left(t_{j}\right)-x\left(t_{j-1}\right)\right)
$$


for $t_{j-1} \leq t \leq t_{j}$. Similarly, for $\vec{\eta}=\left(\eta_{0}, \eta_{1}, \cdots, \eta_{n}\right) \in \mathbb{R}^{n+1}$, define the function $[\vec{\eta}]$ of $\vec{\eta}$ on $[0, T]$ by

$$
[\vec{\eta}](t)=\eta_{j-1}+\frac{t-t_{j-1}}{t_{j}-t_{j-1}}\left(\eta_{j}-\eta_{j-1}\right)
$$

where $t_{j-1} \leq t \leq t_{j}$. Then both $[x]$ and $[\vec{\eta}]$ are continuous on $[0, T]$, they are line segments on each interval $\left[t_{j-1}, t_{j}\right]$, and $[x]\left(t_{j}\right)=x\left(t_{j}\right)$ and $[\vec{\eta}]\left(t_{j}\right)=\eta_{j}$ at each $t_{j}$

Remark 3.1. In [13], the authors presented a simple formula for expressing conditional Wiener integrals in terms of ordinary Wiener integrals. Also, in [3, 16], the authors introduced a simple formula for the analogue of conditional Wiener integral which is essentially similar to Park and Skoug's simple formula on Wiener space; in other words,

$$
E\left[F \mid X_{\tau}\right](\vec{\eta})=\int_{C[0, T]} F(x-[x]+[\vec{\eta}]) d w_{\varphi}(x) .
$$

Equation (3.3) is referred to as the simple formula giving a conditioning function $X_{\tau}$ on the analogue of Wiener space.

The following lemma was established in [3].

Lemma 3.2. Let $t \in\left(t_{j-1}, t_{j}\right)$ for $j=1, \cdots, n$ and $f: \mathbb{R} \rightarrow \mathbb{R}$ be a Borel measurable function. Then

$$
\int_{C[0, T]} f(x(t)-[x](t)) d w_{\varphi}(x) \stackrel{*}{=}\left(\frac{\Phi_{j}(t)}{2 \pi}\right)^{\frac{1}{2}} \int_{\mathbb{R}} f(u) \exp \left\{-\frac{\Phi_{j}(t)}{2} u^{2}\right\} d u
$$

where

$$
\Phi_{j}(t)=\frac{\left(t_{j}-t_{j-1}\right)}{\left(t_{j}-t\right)\left(t-t_{j-1}\right)} .
$$

We present some examples of the analogue of conditional Wiener integral.

Example 3.3. (1) Let $F$ be as in Example 2.1 and let $X_{\tau}$ be given by equation (3.2). Then the analogue of conditional Wiener integral for $F$

$$
\begin{aligned}
& E\left[F \mid X_{\tau}\right](\vec{\eta})=\int_{C[0, T]} \exp \{x(t)-[x](t)+[\vec{\eta}](t)\} d w_{\varphi}(x) \\
& =\left(\frac{T}{2 \pi t(T-t)}\right)^{\frac{1}{2}} \int_{\mathbb{R}} \exp \left\{u+\eta_{0}+\frac{t\left(\eta_{1}-\eta_{0}\right)}{T}\right\} \exp \left\{-\frac{T}{2 t(T-t)} u^{2}\right\} d u \\
& =\exp \left\{\frac{t(T-t)}{2 T}+\eta_{0}+\frac{t\left(\eta_{1}-\eta_{0}\right)}{T}\right\} .
\end{aligned}
$$


(2) Let $F$ be as in Example 2.2 and let $X_{\tau}$ be given by equation (3.2). Then using equation (3.4), we have

$$
\begin{aligned}
& E\left[F \mid X_{\tau}\right](\vec{\eta})=\int_{C[0, T]} \hat{\nu}(x(t)-[x](t)+[\vec{\eta}](t)) d w_{\varphi}(x) \\
& =\left(\frac{T}{2 \pi t(T-t)}\right)^{\frac{1}{2}} \int_{\mathbb{R}} \hat{\nu}\left(u+\eta_{0}+\frac{t\left(\eta_{1}-\eta_{0}\right)}{T}\right) \exp \left\{-\frac{T}{2 t(T-t)} u^{2}\right\} d u \\
& =\left(\frac{T}{2 \pi t(T-t)}\right)^{\frac{1}{2}} \int_{\mathbb{R}}\left[\int_{\mathbb{R}} \exp \left\{i\left(u+\eta_{0}+\frac{t\left(\eta_{1}-\eta_{0}\right)}{T}\right) v\right\} d \nu(v)\right] \\
& =\int_{\mathbb{R}} \exp \left\{-\frac{\exp \left\{-\frac{T}{2 t(T-t)} u^{2}\right\} d u}{2 T} v^{2}+i\left(\eta_{0}+\frac{t\left(\eta_{1}-\eta_{0}\right)}{T}\right) v\right\} d \nu(v) .
\end{aligned}
$$

In our next theorem, we obtain the analogue of conditional Wiener integral for the functional $F$ defined by equation (1.3) above.

Theorem 3.4. Let $F$ be given by equation (3.1) such that $\int_{C[0, T]}|F(x)| d w_{\varphi}(x)<$ $\infty$. Let $X_{\tau}$ be given by equation (3.2). Then the analogue of conditional Wiener integral $E\left[F \mid X_{\tau}\right](\vec{\eta})$ exists for $w_{\varphi} \circ X_{\tau}^{-1}$-a.e. $\vec{\eta} \in \mathbb{R}^{n+1}$ and is given by the formula

$$
\begin{aligned}
& E\left[F \mid X_{\tau}\right](\vec{\eta}) \\
& =1+\sum_{m=1}^{\infty} \sum_{l_{1}+\cdots+l_{n}=m}(-1)^{m} \int_{\prod_{j=1}^{n} \Delta_{\left[t_{j-1}, t_{j}\right), l_{j}}} \prod_{j=1}^{n} \prod_{v=1}^{l_{j}}\left(\frac{\Phi_{j}\left(t_{j-1, v}\right)}{2 \pi}\right)^{\frac{1}{2}} \\
& \quad \cdot \int_{\mathbb{R}} V\left(u+[\vec{\eta}]\left(t_{j-1, v}\right)\right) \exp \left\{-\frac{\Phi_{j}\left(t_{j-1, v}\right)}{2} u^{2}\right\} d u d \prod_{j=1}^{n} \prod_{v=1}^{l_{j}} t_{j-1, v}
\end{aligned}
$$

where $\Delta_{\left[t_{j-1}, t_{j}\right), l_{j}}=\left\{\left(t_{j-1,1}, \cdots, t_{j-1, l_{j}}\right) \mid t_{j-1}<t_{j-1,1}<\cdots<t_{j-1, l_{j}}<t_{j}\right\}$ for $j=1, \cdots, n$ and $t_{0}=0$.

Proof. From our assumption that $F$ is $w_{\varphi}$ integrable, $E\left[F \mid X_{\tau}\right]$ exists for $w_{\varphi} \circ X_{\tau}^{-1}$ a.e. $\vec{\eta} \in \mathbb{R}^{n+1}$. By using equation (3.3) and the Dominated Convergence Theorem, we have

$$
\begin{aligned}
& E\left[F \mid X_{\tau}\right](\vec{\eta}) \\
& =\int_{C[0, T]} \exp \left\{-\int_{0}^{T} V(x(t)-[x](t)+[\vec{\eta}](t)) d t\right\} d w_{\varphi}(x) \\
& =1+\int_{C[0, T]} \sum_{m=1}^{\infty} \frac{(-1)^{m}}{m !}\left(\int_{0}^{T} V(x(t)-[x](t)+[\vec{\eta}](t)) d t\right)^{m} d w_{\varphi}(x) \\
& =1+\sum_{m=1}^{\infty} \int_{C[0, T]} \frac{(-1)^{m}}{m !}\left(\int_{0}^{T} V(x(t)-[x](t)+[\vec{\eta}](t)) d t\right)^{m} d w_{\varphi}(x) .
\end{aligned}
$$


Since $x(t)-[x](t)=\frac{\left(t_{j}-t\right)\left(x(t)-x\left(t_{j-1}\right)\right)}{t_{j}-t_{j-1}}-\frac{\left(t-t_{j-1}\right)\left(x\left(t_{j}\right)-x(t)\right)}{t_{j}-t_{j-1}}$, we have

$$
\begin{aligned}
& E\left[F \mid X_{\tau}\right](\vec{\eta}) \\
& =1+\sum_{m=1}^{\infty} \int_{C[0, T]} \frac{(-1)^{m}}{m !}\left(\sum _ { j = 1 } ^ { n } \int _ { t _ { j - 1 } } ^ { t _ { j } } V \left(\frac{\left(t_{j}-t\right)\left(x(t)-x\left(t_{j-1}\right)\right)}{t_{j}-t_{j-1}}\right.\right. \\
& \left.\left.-\frac{\left(t-t_{j-1}\right)\left(x\left(t_{j}\right)-x(t)\right)}{t_{j}-t_{j-1}}+[\vec{\eta}](t)\right) d t\right)^{m} d w_{\varphi}(x) \\
& \stackrel{(I)}{=} 1+\sum_{m=1}^{\infty} \int_{C[0, T]} \frac{(-1)^{m}}{m !} \sum_{l_{1}+\cdots+l_{n}=m} \frac{m !}{l_{1} ! \cdots l_{n} !} \\
& \cdot\left(\int_{t_{0}}^{t_{1}} V\left(\frac{\left(t_{1}-t\right)\left(x(t)-x\left(t_{0}\right)\right)}{t_{1}-t_{0}}-\frac{\left(t-t_{0}\right)\left(x\left(t_{1}\right)-x(t)\right)}{t_{1}-t_{0}}+[\vec{\eta}](t)\right) d t\right)^{l_{1}} \\
& \cdot\left(\int _ { t _ { n - 1 } } ^ { t _ { n } } V \left(\frac{\left(t_{n}-t\right)\left(x(t)-x\left(t_{n-1}\right)\right)}{t_{n}-t_{n-1}}\right.\right. \\
& \left.\left.-\frac{\left(t-t_{n-1}\right)\left(x\left(t_{n}\right)-x(t)\right)}{t_{n}-t_{n-1}}+[\vec{\eta}](t)\right) d t\right)^{l_{n}} d w_{\varphi}(x) .
\end{aligned}
$$

Step (I) results from the following formula known as the Multinomial Theorem

$$
\left(v_{1}+\cdots+v_{n}\right)^{m}=\sum_{l_{1}+\cdots+l_{n}=m} \frac{m !}{l_{1} ! \cdots l_{n} !} v_{1}^{l_{1}} \cdots v_{n}^{l_{n}}
$$

for any nonnegative integers $l_{1}, \cdots, l_{m}$. Note that for each $j=1,2, \cdots, n$,

$$
\begin{aligned}
& \left(\int_{t_{j-1}}^{t_{j}} V\left(\frac{\left(t_{j}-t\right)\left(x(t)-x\left(t_{j-1}\right)\right)}{t_{j}-t_{j-1}}-\frac{\left(t-t_{j-1}\right)\left(x\left(t_{j}\right)-x(t)\right)}{t_{j}-t_{j-1}}+[\vec{\eta}](t)\right) d t\right)^{l_{j}} \\
& =l_{j} ! \int_{\Delta_{\left[t_{j-1}, t_{j},\right.}, l_{j}} \prod_{v=1}^{l_{j}} V\left(\frac{\left(t_{j}-t_{j-1, v}\right)\left(x\left(t_{j-1, v}\right)-x\left(t_{j-1}\right)\right)}{t_{j}-t_{j-1}}\right. \\
& \left.\quad-\frac{\left(t_{j-1, v}-t_{j-1}\right)\left(x\left(t_{j}\right)-x\left(t_{j-1, v}\right)\right)}{t_{j}-t_{j-1}}+[\vec{\eta}]\left(t_{j-1, v}\right)\right) d \prod_{v=1}^{l_{j}} t_{j-1, v},
\end{aligned}
$$

and the last expression in the above is equal to

$$
\begin{aligned}
& 1+\sum_{m=1}^{\infty} \int_{C[0, T]} \sum_{l_{1}+\cdots+l_{n}=m}(-1)^{m} \\
& \quad\left[\int _ { \prod _ { j = 1 } ^ { n } \Delta _ { [ t _ { j - 1 } , t _ { j } ) , l _ { j } } } \prod _ { j = 1 } ^ { n } \prod _ { v = 1 } ^ { l _ { j } } V \left(\frac{\left(t_{j}-t_{j-1, v}\right)\left(x\left(t_{j-1, v}\right)-x\left(t_{j-1}\right)\right)}{t_{j}-t_{j-1}}\right.\right. \\
& \left.\left.\quad-\frac{\left(t_{j-1, v}-t_{j-1}\right)\left(x\left(t_{j}\right)-x\left(t_{j-1, v}\right)\right)}{t_{j}-t_{j-1}}+[\vec{\eta}]\left(t_{j-1, v}\right)\right) d \prod_{j=1}^{n} \prod_{v=1}^{l_{j}} t_{j-1, v}\right] d w_{\varphi}(x) .
\end{aligned}
$$


Hence, by using Fubini's Theorem and equation (3.4), we have

$$
\begin{aligned}
& E\left[F \mid X_{\tau}\right](\vec{\eta}) \\
& =1+\sum_{m=1}^{\infty} \sum_{l_{1}+\cdots+l_{n}=m}(-1)^{m} \int_{\prod_{j=1}^{n} \Delta_{\left[t_{j-1}, t_{j}\right), l_{j}}} \prod_{j=1}^{n} \prod_{v=1}^{l_{j}}\left(\frac{\Phi_{j}\left(t_{j-1, v}\right)}{2 \pi}\right)^{\frac{1}{2}} \\
& \cdot \int_{\mathbb{R}} V\left(u+[\vec{\eta}]\left(t_{j-1, v}\right)\right) \exp \left\{-\frac{\Phi_{j}\left(t_{j-1, v}\right)}{2} u^{2}\right\} d u d \prod_{j=1}^{n} \prod_{v=1}^{l_{j}} t_{j-1, v} .
\end{aligned}
$$

This proves the desired result.

\section{Applications to Quantum mechanics}

In this section, we use Theorem 3.4 to evaluate the analogue of conditional Wiener integral for functionals involving useful potential functions from quantum mechanics and other areas of physics.

The next two lemmas play a key role in obtaining Theorem 4.3 below.

Lemma 4.1. For $0 \leq s<t \leq T, m \in \mathbb{N}$ and $l_{1}, \cdots, l_{m} \in \mathbb{N}$,

$$
\int_{\mathbb{R}}|v|^{l_{1}+\cdots+l_{m}} \exp \left\{-\frac{v^{2}}{2(t-s)}\right\} d v \leq \sqrt{2 \pi(t-s)}+\int_{\mathbb{R}}|v|^{m L} \exp \left\{-\frac{v^{2}}{2(t-s)}\right\} d v
$$

and if $\varphi$ is a probability measure on $(\mathbb{R}, \mathcal{B}(\mathbb{R}))$, then

$$
\int_{\mathbb{R}}|v|^{l_{1}+\cdots+l_{m}} d \varphi(v) \leq 1+\int_{\mathbb{R}}|v|^{m L} d \varphi(v)
$$

where $L=\max \left\{l_{1}, \cdots, l_{m}\right\}$.

Proof. For $l_{1}, \cdots, l_{m} \in \mathbb{N}$, we have

$$
\begin{aligned}
& \int_{\mathbb{R}}|v|^{l_{1}+\cdots+l_{m}} \exp \left\{-\frac{v^{2}}{2(t-s)}\right\} d v \\
& \leq \int_{|v| \leq 1} 1 \exp \left\{-\frac{v^{2}}{2(t-s)}\right\} d v+\int_{|v|>1}|v|^{m L} \exp \left\{-\frac{v^{2}}{2(t-s)}\right\} d v \\
& \leq \sqrt{2 \pi(t-s)}+\int_{\mathbb{R}}|v|^{m L} \exp \left\{-\frac{v^{2}}{2(t-s)}\right\} d v
\end{aligned}
$$

Similarly, we observe that

$$
\int_{\mathbb{R}}|v|^{l_{1}+\cdots+l_{m}} d \varphi(v) \leq \int_{|v| \leq 1} 1 d \varphi(v)+\int_{|v|>1}|v|^{m L} d \varphi(v) \leq 1+\int_{\mathbb{R}}|v|^{m L} d \varphi(v) .
$$

This proves the desired result.

Lemma 4.2. For each $k \in \mathbb{N}$, let $F_{k}(x)=\exp \left\{-\int_{0}^{T}(x(t))^{k} d t\right\}$ for $x \in C[0, T]$ with

$$
\int_{\mathbb{R}} \exp \left\{M_{0}\left(1+\left|u_{0}\right|^{k}\right)\right\} d \varphi\left(u_{0}\right)<\infty
$$

for some $M_{0}>0$. Then $F_{k}$ is $w_{\varphi}$-integrable for all $k \in \mathbb{N}$. 
Proof. By using series expansion, the Change of Variable Theorem and the BeppoLevi Theorem, it follows that for $k=1,2, \cdots$,

$$
\begin{aligned}
& \int_{C[0, T]}\left|F_{k}(x)\right| d w_{\varphi}(x) \leq 1+\sum_{m=1}^{\infty} \int_{C[0, T]}\left|\frac{1}{m !}\left(-\int_{0}^{T}(x(t))^{k} d t\right)^{m}\right| d w_{\varphi}(x) \\
& =1+\sum_{m=1}^{\infty} \int_{C[0, T]}\left|\frac{(-1)^{m}}{m !}\left(m ! \int_{\Delta_{m}(T)}\left(x\left(t_{1}\right)\right)^{k} \cdots\left(x\left(t_{m}\right)\right)^{k} d t_{1} \cdots d t_{m}\right)\right| d w_{\varphi}(x) \\
& \leq 1+\sum_{m=1}^{\infty} \int_{\Delta_{m}(T)}\left[\int_{C[0, T]}\left|x\left(t_{1}\right)\right|^{k} \cdots\left|x\left(t_{m}\right)\right|^{k} d w_{\varphi}(x)\right] d t_{1} \cdots d t_{m} \\
& =1+\sum_{m=1}^{\infty} \int_{\Delta_{m}(T)}\left[\left(\prod_{j=1}^{m} \frac{1}{2 \pi\left(t_{j}-t_{j-1}\right)}\right)^{\frac{1}{2}} \int_{\mathbb{R}} \int_{\mathbb{R}^{m}}\left|u_{1}\right|^{k} \cdots\left|u_{m}\right|^{k}\right. \\
& \left.\cdot \exp \left\{-\sum_{j=1}^{m} \frac{\left(u_{j}-u_{j-1}\right)^{2}}{2\left(t_{j}-t_{j-1}\right)}\right\} d u_{1} \cdots d u_{m} d \varphi\left(u_{0}\right)\right] d t_{1} \cdots d t_{m}
\end{aligned}
$$


where $\Delta_{m}(T)=\left\{\left(t_{1}, \cdots, t_{m}\right) \mid 0=t_{0}<t_{1}<\cdots<t_{m}=T\right\}$. Next let $u_{j}-u_{j-1}=$ $v_{j}$ for $j=1, \cdots, m$. Then by using equation (3.6),

$$
\begin{aligned}
& \int_{C[0, T]}\left|F_{k}(x)\right| d w_{\varphi}(x) \\
& \leq 1+\sum_{m=1}^{\infty} \int_{\Delta_{m}(T)}\left[\left(\prod_{j=1}^{m} \frac{1}{2 \pi\left(t_{j}-t_{j-1}\right)}\right)^{\frac{1}{2}} \int_{\mathbb{R}} \int_{\mathbb{R}^{m}}\left|v_{1}+u_{0}\right|^{k} \cdots\left|v_{m}+\cdots+v_{1}+u_{0}\right|^{k}\right. \\
& \left.\cdot \exp \left\{-\sum_{j=1}^{m} \frac{v_{j}^{2}}{2\left(t_{j}-t_{j-1}\right)}\right\} d v_{1} \cdots d v_{m} d \varphi\left(u_{0}\right)\right] d t_{1} \cdots d t_{m} \\
& =1+\sum_{m=1}^{\infty} \int_{\Delta_{m}(T)}\left[\left(\prod_{j=1}^{m} \frac{1}{2 \pi\left(t_{j}-t_{j-1}\right)}\right)^{\frac{1}{2}} \int_{\mathbb{R}} \int_{\mathbb{R}^{m}} \sum_{l_{1,1}+l_{1,2}=k} \frac{k !}{l_{1,1} ! l_{1,2} !}\left|v_{1}\right|^{l_{1,1}}\left|u_{0}\right|^{l_{1,2}}\right. \\
& \sum_{l_{2,1}+l_{2,2}+l_{2,3}=k} \frac{k !}{l_{2,1} ! l_{2,2} ! l_{2,3} !}\left|v_{2}\right|^{l_{2,1}}\left|v_{1}\right|^{l_{2,2}}\left|u_{0}\right|^{l_{2,3}} \\
& \sum_{l_{m, 1}+l_{m, 2}+\cdots+l_{m, m+1}=k} \frac{k !}{l_{m, 1} ! l_{m, 2} ! \cdots l_{m, m} ! l_{m, m+1} !}\left|v_{m}\right|^{l_{m, 1}} \cdots\left|v_{1}\right|^{l_{m, m}}\left|u_{0}\right|^{l_{m, m+1}} \\
& \left.\cdot \exp \left\{-\sum_{j=1}^{m} \frac{v_{j}^{2}}{2\left(t_{j}-t_{j-1}\right)}\right\} d v_{1} \cdots d v_{m} d \varphi\left(u_{0}\right)\right] d t_{1} \cdots d t_{m} \\
& =1+\sum_{m=1}^{\infty} \int_{\Delta_{m}(T)}\left[\left(\prod_{j=1}^{m} \frac{1}{2 \pi\left(t_{j}-t_{j-1}\right)}\right)^{\frac{1}{2}} \sum_{l_{1,1}+l_{1,2}=k} \sum_{l_{2,1}+l_{2,2}+l_{2,3}=k} \ldots \sum_{l_{m, 1}+\cdots+l_{m, m+1}=k}\right. \\
& \cdot\left(\frac{k !}{l_{1,1} ! l_{1,2} !}\right)\left(\frac{k !}{l_{2,1} ! l_{2,2} ! l_{2,3} !}\right) \cdots\left(\frac{k !}{l_{m, 1} ! l_{m, 2} ! \cdots l_{m, m} ! l_{m, m+1} !}\right) \\
& \cdot \int_{\mathbb{R}}\left|v_{1}\right|^{l_{1,1}+l_{2,2}+\cdots+l_{m, m}} \exp \left\{-\frac{v_{1}^{2}}{2\left(t_{1}-t_{0}\right)}\right\} d v_{1} \\
& \cdot \int_{\mathbb{R}}\left|v_{2}\right|^{l_{2,1}+l_{3,2}+l_{4,3}+\cdots+l_{m, m-1}} \exp \left\{-\frac{v_{2}^{2}}{2\left(t_{2}-t_{1}\right)}\right\} d v_{2} \\
& \cdot \int_{\mathbb{R}}\left|v_{m}\right|^{l_{m, 1}} \exp \left\{-\frac{v_{m}^{2}}{2\left(t_{m}-t_{m-1}\right)}\right\} d v_{m} \\
& \left.\cdot \int_{\mathbb{R}}\left|u_{0}\right|^{l_{1,2}+l_{2,3}+l_{3,4}+\cdots+l_{m, m+1}} d \varphi\left(u_{0}\right)\right] d t_{1} \cdots d t_{m} .
\end{aligned}
$$

Applying Lemma 4.1 to the last expression in the above equation, we obtain

$$
\begin{aligned}
& \int_{C[0, T]}\left|F_{k}(x)\right| d w_{\varphi}(x) \\
& \leq 1+\sum_{m=1}^{\infty} \int_{\Delta_{m}(T)}\left[\left(\prod_{j=1}^{m} \frac{1}{2 \pi\left(t_{j}-t_{j-1}\right)}\right)^{\frac{1}{2}} \sum_{l_{1,1}+l_{1,2}=k} \sum_{l_{2,1}+l_{2,2}+l_{2,3}=k} \ldots \sum_{l_{m, 1}+\cdots+l_{m, m+1}=k}\right.
\end{aligned}
$$




$$
\begin{aligned}
& \cdot\left(\frac{k !}{l_{1,1} ! l_{1,2} !}\right)\left(\frac{k !}{l_{2,1} ! l_{2,2} ! l_{2,3} !}\right) \cdots\left(\frac{k !}{l_{m, 1} ! \cdots l_{m, m+1} !}\right) \\
& \cdot\left(\sqrt{2 \pi\left(t_{1}-t_{0}\right)}+\int_{\mathbb{R}}\left|v_{1}\right|^{k m} \exp \left\{-\frac{v_{1}^{2}}{2\left(t_{1}-t_{0}\right)}\right\} d v_{1}\right) \\
& \cdot\left(\sqrt{2 \pi\left(t_{2}-t_{1}\right)}+\int_{\mathbb{R}}\left|v_{2}\right|^{k m} \exp \left\{-\frac{v_{2}^{2}}{2\left(t_{2}-t_{1}\right)}\right\} d v_{2}\right) \\
& \cdots\left(\sqrt{2 \pi\left(t_{m}-t_{m-1}\right)}+\int_{\mathbb{R}}\left|v_{m}\right|^{k m} \exp \left\{-\frac{v_{m}^{2}}{2\left(t_{m}-t_{m-1}\right)}\right\} d v_{m}\right) \\
& \left.\cdot\left(1+\int_{\mathbb{R}}\left|u_{0}\right|^{k m} d \varphi\left(u_{0}\right)\right)\right] d t_{1} \cdots d t_{m} .
\end{aligned}
$$

Since $\sum_{l_{m, 1}+\cdots+l_{m, m+1}=k} \frac{k !}{l_{m, 1} ! \cdots l_{m, m+1} !}=(m+1)^{k}$, for all $m \in \mathbb{N}$, we have

$$
\begin{aligned}
& \int_{C[0, T]}\left|F_{k}(x)\right| d w_{\varphi}(x) \\
& \leq 1+\sum_{m=1}^{\infty} \int_{\Delta_{m}(T)}\left[\left(2^{k}+\left(\frac{1}{2 \pi\left(t_{1}-t_{0}\right)}\right)^{\frac{1}{2}} \int_{\mathbb{R}}\left|v_{1}\right|^{k m} \exp \left\{-\frac{v_{1}^{2}}{2\left(t_{1}-t_{0}\right)}\right\} d v_{1}\right)\right. \\
& \cdot\left(3^{k}+\left(\frac{1}{2 \pi\left(t_{2}-t_{1}\right)}\right)^{\frac{1}{2}} \int_{\mathbb{R}}\left|v_{2}\right|^{k m} \exp \left\{-\frac{v_{2}^{2}}{2\left(t_{2}-t_{1}\right)}\right\} d v_{2}\right) \\
& \quad \cdot\left((m+1)^{k}+\left(\frac{1}{2 \pi\left(t_{m}-t_{m-1}\right)}\right)^{\frac{1}{2}} \int_{\mathbb{R}}\left|v_{m}\right|^{k m} \exp \left\{-\frac{v_{m}^{2}}{2\left(t_{m}-t_{m-1}\right)}\right\} d v_{m}\right) \\
& \left.\cdot\left(1+\int_{\mathbb{R}}\left|u_{0}\right|^{k m} d \varphi\left(u_{0}\right)\right)\right] d t_{1} \cdots d t_{m} .
\end{aligned}
$$

Now, let $M=\max \left\{M_{1}^{k}, \cdots, M_{m}^{k}\right\}$ where

$$
M_{j}^{k}=\left((m+1)^{k}+\left(\frac{1}{2 \pi\left(t_{j}-t_{j-1}\right)}\right)^{\frac{1}{2}} \int_{\mathbb{R}}\left|v_{j}\right|^{k m} \exp \left\{-\frac{v_{j}^{2}}{2\left(t_{j}-t_{j-1}\right)}\right\} d v_{j}\right)
$$

for $j=1, \cdots, m$ and for all $k \in \mathbb{N}$. Then using equation (4.1) and the Beppo-Levi Theorem, we have

$$
\begin{aligned}
\int_{C[0, T]}\left|F_{k}(x)\right| d w_{\varphi}(x) & \leq 1+\sum_{m=1}^{\infty} \int_{\Delta_{m}(T)} M^{m}\left(\int_{\mathbb{R}}\left(1+\left|u_{0}\right|^{k}\right)^{m} d \varphi\left(u_{0}\right)\right) d t_{1} \cdots d t_{m} \\
& =1+\int_{\mathbb{R}} \sum_{m=1}^{\infty}\left(M\left(1+\left|u_{0}\right|^{k}\right)\right)^{m} \int_{\Delta_{m}(T)} d t_{1} \cdots d t_{m} d \varphi\left(u_{0}\right) \\
& =1+\int_{\mathbb{R}} \sum_{m=1}^{\infty}\left(M\left(1+\left|u_{0}\right|^{k}\right)\right)^{m} \frac{1}{m !}\left(\int_{0}^{T} 1 d t\right)^{m} d \varphi\left(u_{0}\right) \\
& =\int_{\mathbb{R}} \exp \left\{M_{0}\left(1+\left|u_{0}\right|^{k}\right)\right\} d \varphi\left(u_{0}\right)<\infty,
\end{aligned}
$$


where $M_{0}=M T$. This proves the desired result.

In our next theorem, as an application of Theorem 3.4, we express the analogue of conditional Wiener integral $E\left[F_{k} \mid X_{\tau}\right]$ in terms of the potential function $V(u)=$ $u^{k}(k \in \mathbb{N})$.

Theorem 4.3. Let $F_{k}$ be as in Lemma 4.2 and let $X_{\tau}$ be given by equation (3.2). Then the analogue of conditional Wiener integral $E\left[F_{k} \mid X_{\tau}\right](\vec{\eta})$ exists for $w_{\varphi} \circ X_{\tau}^{-1}$-a.e. $\vec{\eta} \in \mathbb{R}^{n+1}$ and is given by the formula

$$
E\left[F_{k} \mid X_{\tau}\right](\vec{\eta})=\exp \left\{-\sum_{j=1}^{n} \int_{t_{j-1}}^{t_{j}} \sum_{l=0}^{\left[\frac{k}{2}\right]} \frac{k !}{(k-2 l) ! 2^{l} l !}([\vec{\eta}](t))^{k-2 l}\left(\frac{1}{\Phi_{j}(t)}\right)^{l} d t\right\}
$$

where $\Phi_{j}(t)$ is given by equation (3.5).

Proof. In Lemma 4.2, we have shown that $F_{k}$ is $w_{\varphi}$-integrable and so by Theorem 3.4, $E\left[F_{k} \mid X_{\tau}\right]$ exists for $w_{\varphi} \circ X_{\tau}^{-1}$-a.e. $\vec{\eta} \in \mathbb{R}^{n+1}$ and for all $k=1,2, \cdots$. Now we evaluate the analogue of conditional Wiener integral. Letting $V(u)=u^{k}$ for $k \in \mathbb{N}$ and applying Theorem 3.4, we have

$$
\begin{aligned}
& E\left[F_{k} \mid X_{\tau}\right](\vec{\eta}) \\
& =1+\sum_{m=1}^{\infty} \sum_{l_{1}+\cdots+l_{n}=m}(-1)^{m} \int_{\prod_{j=1}^{n} \Delta_{\left[t_{j-1}, t_{j}\right), l_{j}}} \prod_{j=1}^{n} \prod_{v=1}^{l_{j}}\left(\frac{\Phi_{j}\left(t_{j-1, v}\right)}{2 \pi}\right)^{\frac{1}{2}} \\
& \cdot \int_{\mathbb{R}}\left(u+[\vec{\eta}]\left(t_{j-1, v}\right)\right)^{k} \exp \left\{-\frac{\Phi_{j}\left(t_{j-1, v}\right)}{2} u^{2}\right\} d u d \prod_{j=1}^{n} \prod_{v=1}^{l_{j}} t_{j-1, v}
\end{aligned}
$$

where $\Phi_{j}\left(t_{j-1, v}\right)$ is given by equation (3.5). Next using the Binomial Theorem, we obtain

$$
\begin{aligned}
E\left[F_{k} \mid X_{\tau}\right](\vec{\eta})=1+\sum_{m=1}^{\infty} \sum_{l_{1}+\cdots+l_{n}=m}(-1)^{m} \int_{\prod_{j=1}^{n} \Delta_{\left[t_{j-1}, t_{j}, l_{j}\right.}} \prod_{j=1}^{n} \prod_{v=1}^{l_{j}}\left(\frac{\Phi_{j}\left(t_{j-1, v}\right)}{2 \pi}\right)^{\frac{1}{2}} \\
\cdot \sum_{l=0}^{k}\left(\begin{array}{c}
k \\
l
\end{array}\right)\left([\vec{\eta}]\left(t_{j-1, v}\right)\right)^{k-l} \int_{\mathbb{R}} u^{l} \exp \left\{-\frac{\Phi_{j}\left(t_{j-1, v}\right)}{2} u^{2}\right\} d u d \prod_{j=1}^{n} \prod_{v=1}^{l_{j}} t_{j-1, v} \\
=1+\sum_{m=1}^{\infty} \sum_{l_{1}+\cdots+l_{n}=m}(-1)^{m} \int_{\prod_{j=1}^{n} \Delta_{\left[t_{j-1}, t_{j}\right), l_{j}}} \prod_{j=1}^{n} \prod_{v=1}^{l_{j}}\left(\frac{\Phi_{j}\left(t_{j-1, v}\right)}{2 \pi}\right)^{\frac{1}{2}} \\
\cdot 2 \sum_{l=0}^{\left[\frac{k}{2}\right]}\left(\begin{array}{c}
k \\
2 l
\end{array}\right)\left([\vec{\eta}]\left(t_{j-1, v}\right)\right)^{k-2 l} \int_{0}^{\infty} u^{2 l} \exp \left\{-\frac{\Phi_{j}\left(t_{j-1, v}\right)}{2} u^{2}\right\} d u d \prod_{j=1}^{n} \prod_{v=1}^{l_{j}} t_{j-1, v} .
\end{aligned}
$$

Let $w=\frac{\Phi_{j}\left(t_{j-1, v}\right)}{2} u^{2}$. Then $\int_{0}^{\infty} w^{\frac{2 l+1}{2}-1} \exp \{-w\} d w=\Gamma\left(\frac{2 l+1}{2}\right)$ and so

$$
\int_{0}^{\infty} u^{2 l} \exp \left\{-\frac{\Phi_{j}\left(t_{j-1, v}\right)}{2} u^{2}\right\} d u=\frac{1}{2}\left(\frac{2}{\Phi_{j}\left(t_{j-1, v}\right)}\right)^{l}\left(\frac{2}{\Phi_{j}\left(t_{j-1, v}\right)}\right)^{\frac{1}{2}} \Gamma\left(\frac{2 l+1}{2}\right)
$$


where $\Gamma$ is the Gamma function. Applying this to the last expression in equation (4.2), we obtain

$$
\begin{aligned}
& E\left[F_{k} \mid X_{\tau}\right](\vec{\eta}) \\
& =1+\sum_{m=1}^{\infty} \sum_{l_{1}+\cdots+l_{n}=m}(-1)^{m} \int_{\prod_{j=1}^{n} \Delta_{\left[t_{j-1}, t_{j}\right), l_{j}}} \prod_{j=1}^{n} \prod_{v=1}^{l_{j}}\left(\frac{1}{\pi}\right)^{\frac{1}{2}} \\
& \quad \cdot \sum_{l=0}^{\left[\frac{k}{2}\right]}\left(\begin{array}{c}
k \\
2 l
\end{array}\right)\left([\vec{\eta}]\left(t_{j-1, v}\right)\right)^{k-2 l} \Gamma\left(\frac{2 l+1}{2}\right)\left(\frac{2}{\Phi_{j}\left(t_{j-1, v}\right)}\right)^{l} d \prod_{j=1}^{n} \prod_{v=1}^{l_{j}} t_{j-1, v} \\
& =1+\sum_{m=1}^{\infty} \sum_{l_{1}+\cdots+l_{n}=m}(-1)^{m} \int_{\prod_{j=1}^{n} \Delta_{\left[t_{j-1}, t_{j}\right), l_{j}}}^{l_{j}} \prod_{j=1} \prod_{v=1} \cdot \sum_{l=0}^{\left[\frac{k}{2}\right]} \frac{k !}{(k-2 l) ! 2^{l} l !}\left([\vec{\eta}]\left(t_{j-1, v}\right)\right)^{k-2 l}\left(\frac{1}{\Phi_{j}\left(t_{j-1, v}\right)}\right)^{l} d \prod_{j=1}^{n} \prod_{v=1}^{l_{j}} t_{j-1, v} \\
& =\exp \left\{-\sum_{j=1}^{n} \int_{t_{j-1}}^{t_{j}} \sum_{l=0}^{\left[\frac{k}{2}\right]} \frac{k !}{(k-2 l) ! 2^{l} l !}([\vec{\eta}](t))^{k-2 l}\left(\frac{1}{\Phi_{j}(t)}\right)^{l} d t\right\}
\end{aligned}
$$

which completes the proof of Theorem 4.3.

In our next example, we apply the results obtained in Theorem 4.3 to the following three special cases.

\begin{tabular}{l|c}
\hline & $E\left[F_{j} \mid X_{\tau}\right](\vec{\eta})(j=1,2,3)$ \\
\hline$F_{1}(x)=\exp \left\{-\int_{0}^{T}(x(t)) d t\right\}$ & $\exp \left\{-\frac{1}{2} \sum_{j=1}^{n}\left(t_{j}-t_{j-1}\right)\left(\eta_{j}+\eta_{j-1}\right)\right\}$ \\
\hline$F_{2}(x)=\exp \left\{-\int_{0}^{T}(x(t))^{2} d t\right\}$ & $\exp \left\{-\frac{1}{6} \sum_{j=1}^{n}\left(t_{j}-t_{j-1}\right)\right.$ \\
\hline$F_{3}(x)=\exp \left\{-\int_{0}^{T}(x(t))^{3} d t\right\}$ & $\left.\cdot\left(t_{j}-t_{j-1}+2 \eta_{j}^{2}+2 \eta_{j} \eta_{j-1}+2 \eta_{j-1}^{2}\right)\right\}$ \\
\hline $\exp \left\{-\frac{1}{4} \sum_{j=1}^{n}\left(t_{j}-t_{j-1}\right)\left[\left(t_{j}-t_{j-1}\right)\left(\eta_{j}+\eta_{j-1}\right)\right.\right.$ \\
$\left.\left.+\eta_{j}^{3}+\eta_{j}^{2} \eta_{j-1}+\eta_{j} \eta_{j-1}^{2}+\eta_{j-1}^{3}\right]\right\}$
\end{tabular}

TABLE 1. 
We now apply Theorems 3.4 and 4.3 to three potential functions. Functions such as these are very important in quantum mechanics and have appeared in other areas of mathematical physics involving various Feynman integration theories.

\section{[1] Harmonic oscillator}

When $V(u)=a^{2} u^{2}, a \in \mathbb{R}-\{0\}$ in equation (1.1), this equation is called the diffusion equation for harmonic oscillator

$$
\frac{\partial}{\partial t} \psi(u, t)=\frac{1}{2} \frac{\partial^{2}}{\partial u^{2}} \psi(u, t)-a^{2} u^{2} \psi(u, t)
$$

with the initial condition $\psi(u, 0)=\varphi(u)$. In this case, the functional $F$ in equation (1.3) becomes

\section{[2] Double-well potential}

$$
F(x)=\exp \left\{-a^{2} \int_{0}^{T} x^{2}(t) d t\right\}
$$

For positive real constants $m, a$, and $\omega$, let $V(u)=\frac{m \omega^{2}}{8 a^{2}}\left(u^{2}-a^{2}\right)^{2}$. This is a double-well potential with minima at $u= \pm a$ [15]. For sufficiently large $a$, by expanding the potential at $u= \pm a$, one can obtain following expression

$$
V(u)=\frac{m \omega^{2}}{2}(u \mp a)^{2}\left[1 \pm \frac{u \mp a}{a}+\frac{(u \mp a)^{2}}{4 a^{2}}\right] \simeq \frac{m \omega^{2}}{2}(u \mp a)^{2} .
$$

When $V(u)=\frac{m \omega^{2}}{8 a^{2}}\left(u^{2}-a^{2}\right)^{2}, m, a, \omega \in \mathbb{R}^{+}$in equation (1.1), this equation is called the diffusion equation for a double-well potential with minima at $u= \pm a$

$$
\frac{\partial}{\partial t} \psi(u, t)=\frac{1}{2} \frac{\partial^{2}}{\partial u^{2}} \psi(u, t)-\frac{m \omega^{2}}{8 a^{2}}\left(u^{2}-a^{2}\right)^{2} \psi(u, t)
$$

with the initial condition $\psi(u, 0)=\varphi(u)$. In this case, the functional $F$ in equation (1.3) becomes

$$
F(x)=\exp \left\{-\frac{m \omega^{2}}{8 a^{2}} \int_{0}^{T}\left(x^{2}(t)-a^{2}\right)^{2} d t\right\} .
$$

\section{[3] Pöschl-Teller potential}

When $V(u)=-\frac{\alpha^{2} h}{2 k} \frac{s(s+1)}{\cosh ^{2}(\alpha u)}$ for $s=1,2, \cdots$, in equation (1.1), this equation is called the diffusion equation for the Pöschl-Teller potential

$$
\frac{\partial}{\partial t} \psi(u, t)=\frac{1}{2} \frac{\partial^{2}}{\partial u^{2}} \psi(u, t)-\frac{\alpha^{2} h}{2 k} \frac{s(s+1)}{\cosh ^{2}(\alpha u)} \psi(u, t)
$$

with the initial condition $\psi(u, 0)=\varphi(u)$. In this case, the functional $F$ in equation (1.3) becomes

$$
F(x)=\exp \left\{\frac{\alpha^{2} h}{2 k} s(s+1) \int_{0}^{T} \frac{1}{\cosh ^{2}(\alpha x(t))} d t\right\} .
$$


We finish this paper by applying Theorem 3.4 for the three potential functions(Equations (4.3), (4.4), (4.5)). The results are summarized in the following;

\begin{tabular}{|c|c|}
\hline & $E\left[F \mid X_{\tau}\right](\vec{\eta})$ \\
\hline Harmonic oscillator & $\begin{aligned} \exp \{- & \frac{a^{2}}{6} \sum_{j=1}^{n}\left(t_{j}-t_{j-1}\right) \\
& \left.\quad\left(t_{j}-t_{j-1}+2 \eta_{j}^{2}+2 \eta_{j} \eta_{j-1}+2 \eta_{j-1}^{2}\right)\right\}\end{aligned}$ \\
\hline Double-well potential & $\begin{array}{l}\exp \left\{-\sum_{j=1}^{n}\left[\frac{m \omega^{2}}{8 a^{2}}\left(\frac{1}{60}\left(t_{j}-t_{j-1}\right)^{3}\right.\right.\right. \\
+\frac{1}{10}\left(t_{j}-t_{j-1}\right)^{2}\left(3 \eta_{j-1}^{2}+4 \eta_{j} \eta_{j-1}+3 \eta_{j}^{2}\right) \\
\left.+\frac{1}{5}\left(t_{j}-t_{j-1}\right)\left(\eta_{j-1}^{4}+\eta_{j-1}^{3} \eta_{j}+\eta_{j-1}^{2} \eta_{j}^{2}+\eta_{j-1} \eta_{j}^{3}+\eta_{j}^{4}\right)\right) \\
+\frac{m \omega^{2} a^{2}}{8}\left(t_{j}-t_{j-1}\right)-\frac{m \omega^{2}}{4}\left(\frac{1}{6}\left(t_{j}-t_{j-1}\right)\right. \\
\left.\left.\quad \cdot\left(t_{j}-t_{j-1}+2 \eta_{j-1}^{2}+2 \eta_{j-1} \eta_{j}+2 \eta_{j}^{2}\right)\right]\right\}\end{array}$ \\
\hline Pöschl-Teller potential & $\begin{aligned} & 1+\sum_{m=1}^{\infty} \sum_{l_{1}+\cdots+l_{n}=m} \int_{\prod_{j=1}^{n} \Delta_{\left[t_{j-1}, t_{j}\right), l_{j}}} \cdot \prod_{j=1}^{n} \prod_{v=1}^{l_{j}}\left(\frac{\alpha^{2} h}{2 k} s(s+1)\right) \\
& \cdot\left[\sum_{w=0}^{\infty} \sum_{q_{0}+\cdots+q_{2 w}=2} \frac{2 !}{q_{0} ! \cdots q_{2 w} !}\left(E_{0}\right)^{q_{0}} \cdots\left(\frac{E_{2 w}}{(2 w) !}\right)^{q_{2 w}}\right. \\
& \cdot \sum_{l=0}^{\left[\frac{Q}{2}\right]} \frac{Q !}{(Q-2 l) ! 2 l ! !}\left(\alpha[\vec{\eta}]\left(t_{j-1, v}\right)\right)^{Q-2 l} \alpha^{2 l} \\
&\left.\quad\left(\frac{1}{\Phi_{j}\left(t_{j-1, v}\right)}\right)^{l}\right] d \prod_{j=1}^{n} \prod_{v=1}^{l_{j}} t_{j-1, v}\end{aligned}$ \\
\hline
\end{tabular}

TABLE 2. Analogue of conditional Wiener integrals

In Table 2 above, $\Phi_{j}\left(t_{j-1, v}\right)$ is given by equation (3.5), and $E_{2 w}$ and $Q$ are given by equation (4.6) below.

Remark 4.4. In Table 2 above, to obtain the analogue of conditional Wiener integral for Pöschl-Teller potential functional $F$ given by equation (4.5), we use the following formula

$$
\begin{aligned}
& \int_{\mathbb{R}} \operatorname{Sech}^{2}(u) \exp \left\{-u^{2}\right\} d u \\
& =\sum_{w=0}^{\infty} \sum_{q_{0}+\cdots+q_{2 w}=2} \frac{2 !}{q_{0} ! \cdots q_{2 w} !}\left(\frac{E_{0}}{0 !}\right)^{q_{0}}\left(\frac{E_{2}}{2 !}\right)^{q_{2}} \cdots\left(\frac{E_{2 w}}{(2 w) !}\right)^{q_{2 w}} \int_{\mathbb{R}} u^{Q} \exp \left\{-u^{2}\right\} d u
\end{aligned}
$$


where $Q=2 q_{2}+\cdots+2 w q_{2 w}$ and $E_{2 w}$ is the Euler number(odd-indexed Euler numbers are all zero); in other words,

$$
E_{2 w}=i \sum_{k=1}^{2 w+1} \sum_{j=0}^{k}\left(\begin{array}{l}
k \\
j
\end{array}\right) \frac{(-1)^{j}(k-2 j)^{2 w+1}}{2^{k} i^{k} k} .
$$

Equation (4.6) follows from the Taylor series expansion of the hyperbolic secant and the $\aleph_{0}$-nominal formula [10, p.441].

Acknowledgement. The authors thank the referees for their helpful suggestions which led to the present version of this paper. The present research was conducted by the research fund of Dankook University in 2014.

\section{REFERENCES}

1. S. Albeverio, Z. Brzezniak and Z. Haba, On the Schrödinger equation with potentials which are Laplace transforms of measures, Potential Anal. 9 (1998), 65-82.

2. S. Albeverio, A. Khrennikov and O. Smolyanov, The probabilistic Feynman-Kac formula for an infinite-dimensional Schrödinger equation with exponential and singular potentials, Potential Anal. 11 (1999), 157-181.

3. D.H. Cho, A simple formula for an analogue of conditional Wiener integrals and its applications, Trans. Amer. Math. Soc. 135 (2008), 1-16.

4. __ A simple formula for an analogue of conditional Wiener integrals and its applications II, Czechoslovak Math. J. 59(134) (2009), 431-452.

5. Conditional Feynman integral and Schrödinger integral equation on a function space, Bull. Aust. Math. Soc. 79 (2009), 1-22.

6 .___Evaluation formulas for an analogue of conditional analytic Feynman integrals over a function space, Bull. Korean Math. Soc. 48 (2011), 655-672.

7. H.S. Chung, J.G. Choi and S.J. Chang, The approach to solution of the Schrödinger equation using Fourier-type functionals, J. Korean Math. Soc. 50 (2013), 259-274.

8. H.S. Chung and S.J. Chang, Some application of the spectral theory for the integral transform involving the spectral representation, J. Func. Space Appl. 2012, Article ID 573602, $17 \mathrm{pp}$.

9. H.S. Chung, and V.K. Tuan, Fourier-type functionals on Wiener space, Bull. Korean Math. Soc. 49 (2012), 609-619.

10. G.W. Johnson and M.L. Lapidus, The Feynman integral and Feynman's operational calculus, Oxford Mathematical Monographs, Clarendon Press, Oxford, 2000.

11. I.Y. Lee, H.S. Chung and S.J. Chang, Series expansions of the analytic Feynman integral for the Fourier-type functional, J. Korean Soc. Math. Educ. Ser. B. 19 (2012), 87-102.

12. K.R. Parthasarathy, Probability measures on metric spaces, Academic Press, New York, 1967.

13. C. Park and D. Skoug, A simple formula for conditional Wiener integrals with applications, Pacific J. Math. 135 (1988), 381-394.

14. C.S. Park, Generalized WKB connection formulas and a symmetrical double-well potential, J. Korean Phys. Soc. 42 (2003), 830-834.

15. C.S. Park, M.G. Jeong, S.K. Yoo, and D.K. Park, Double-well potential: The WKB approximation with phase loss and anharmonicity effect, Phys. Rev. A 58 (1998), 3443-3447.

16. K.S. Ryu, The simple formula of conditional expectation on analogue of Wiener measure, Honam Mathematical J. 4 (2008), 723-732.

17. __ Integration with respect to analogue of Wiener measure over paths in abstract Wiener space and its applications, Bull. Korean Math. Soc. 47 (2010), 131-149.

18. K.S. Ryu and M.K. Im, A measure-valued analogue of Wiener measure and the measurevalued Feynman-Kac formula, Trans. Amer. Math. Soc. 354 (2002), 4921-4951. 
19. An analogue of Wiener measure and its applications, J. Korean Math. Soc. 39 (2002), 801-819.

20. K.S. Ryu and S.H. Shim, The rotation theorem on analogue of Wiener space, Honam Math. J. 29 (2007), 577-588.

21. T. Zastawniak, The equivalence of two approaches to the Feynman integral for the anharmonic oscillator, Univ. Iagel. Acta Math. No. 28 (1991), 187-199.

Department of Mathematics, Dankook University, Cheonan 330-714, Korea.

E-mail address: iylee@dankook.ac.kr

E-mail address: hschung@dankook.ac.kr

E-mail address: sejchang@dankook.ac.kr 